\title{
Cristina Nanni and Stefano Fanti (eds): PET-CT: Rare Findings and Diseases
}

\author{
Springer Verlag, Berlin Heidelberg, 2012. ISBN: 978-3-642-24698-2
}

\author{
Luigi Mansi • Christian Marsiglia
}

Published online: 30 May 2012

(C) Springer-Verlag 2012

In the past 10 years the use of PET/CT in the diagnostic process in oncological patients has rapidly increased, and the indications for the examination have widened. This trend has been accompanied by new therapies, which are significantly increasing patients' life expectancy, yielding a prominent benefit in a number of oncological patients with important comorbidities that may be positive on the $\mathrm{PET} / \mathrm{CT}$ scan. Furthermore, functional imaging is also emerging for the evaluation of patients with inflammatory/infective diseases to guide clinicians in the characterization, identification and localization of the inflammatory areas. As a consequence, nuclear physicians are more and more frequently asked to participate in the diagnostic process in patients affected by rare diseases, in which practical experience may still be poor. In addition, the continually increasing number of PET/CT scans performed have resulted in a higher probability of rare collateral findings which are sometimes hardly recognized or interpreted. Last but not least, the wider use of non-FDG PET tracers (such as ${ }^{11} \mathrm{C}$-choline, ${ }^{11} \mathrm{C}$-methionine and ${ }^{68} \mathrm{Ga}$-somatostatin analogues) has enlarged the spectrum of rare diseases and rare findings met by the nuclear physician in routine practice, creating a difficult challenge particularly for beginners.

L. Mansi $(\bowtie) \cdot$ C. Marsiglia

Medicina Nucleare, Seconda Università di Napoli,

Napoli, Italy

e-mail: luigi.mansi@unina2.it
This unique atlas has collected a large number of clinical cases to help PET/CT users in their routine practice. The cases are divided into two groups: patients evaluated for rare diseases (rare, but frequent enough to be met in clinical practice), and patients evaluated for standard indications for PET/CT whose PET scan is characterized by atypical collateral findings.

The book is organized into seven chapters, of which six, two for each radiotracer, concern rare findings and rare diseases studied with FDG, ${ }^{11} \mathrm{C}$-choline and ${ }^{68} \mathrm{Ga}$-somatostatin analogues. The last chapter presents rare diseases detected with ${ }^{11} \mathrm{C}$-methionine. In this way a total of 136 cases are analysed, with more than 400 pictures. Each case is presented by describing the clinical history of the patient, the PET/CT findings, supported by representative images, a brief description of the disease, and by significant references from the literature when needed.

The style of the atlas is very didactic. Its iconographic quality, simplicity, clarity and completeness of information are in continuity with the three previous successful atlases by Stefano Fanti and published by Springer.

We recommend this book to all who want to improve their knowledge on less frequent diseases evaluated with FDG or radiotracers beyond FDG. The experience of the authors is so extensive that they are able to present diagnostic problems rarely encountered in the majority of PET centres. Therefore this book will be of interest both to experts and beginners, i.e. to physicians and residents who want to improve their knowledge taking advantage of a wide experience.

This atlas provides a practical support in routine practice, and will help change many PET/CT reports from inconclusive to conclusive. 